\title{
FGF-receptor substrate 2 functions as a molecular sensor integrating external regulatory signals into the FGF pathway
}

\author{
Wenchao Zhou ${ }^{1}$, Xiujing Feng ${ }^{1}$, Yingjie $\mathrm{Wu}^{2}$, Johannes Benge ${ }^{3}$, Zhe Zhang ${ }^{1}$, Zhengjun Chen ${ }^{1}$ \\ ${ }^{I}$ State Key Laboratory of Molecular Biology, Institute of Biochemistry and Cell Biology, Shanghai Institutes for Biological Scienc- \\ es, Graduate University of the Chinese Academy of Sciences, Chinese Academy of Sciences, 320 Yueyang Road, Shanghai 200031, \\ China; ${ }^{2}$ Innovation Center China, AstraZeneca Global R\&D, AstraZeneca Pharmaceutical Co, Ltd, Shanghai 200041, China; ${ }^{3}$ U \\ Pharma AG, Bunsenstrasse 1, 82152 Martinsried, Germany
}

Fibroblast growth factor (FGF) receptor substrate $2 \alpha$ (FRS2 $\alpha$ ) is the main mediator of signaling in the FGF pathway. Recent studies have shown that mitogen-activated protein kinase (MAPK) phosphorylates serine and threonine residues in FRS2, negatively affecting FGF-induced tyrosine phosphorylation (PY) of FRS2. Several kinds of stimuli can induce serine/threonine phosphorylation (PS/T) of FRS2, indicating that FRS2 may be useful for studying crosstalk between growth factor signaling pathways. Here, we report that FGF-induced PY of FRS2 can be attenuated by EGF co-stimulation in PC12 cells; this inhibitory effect could be completely reversed by U0126, an inhibitor of MEK. We further identified the ERK1/2-binding motif in FRS2 and generated FRS2-3KL, a mutant lacking MAPK binding and PT upon FGF and/or EGF stimulation. Unlike wild-type (WT) FRS2, FGF-induced PY of FRS2-3KL could not be inhibited by EGF co-stimulation, and FRS2-3KL-expressing PC12 cells exhibited more differentiating potential than FRS2-WT-expressing cells in response to FGF treatment. These results suggest that PS/T of FRS2 mediated by the FRS2-MAPK negative regulatory loop may function as a molecular switch integrating negative regulatory signals from other pathways into FGFR-generated signal transduction.

Keywords: fibroblast growth factor (FGF), epithelial growth factor (EGF), crosstalk, threonine phosphorylation, co-stimulation

Cell Research (2009) 19:1165-1177. doi: 10.1038/cr.2009.95; published online 4 August 2009

\section{Introduction}

Fibroblast growth factor (FGF) receptor substrate 2 (FRS2), an adapter protein, has been identified as a major downstream mediator of FGFR signaling. It is thought to play a critical role in early embryogenesis because knocking out the FRS2 gene in mice leads to embryonic lethality at E7-7.5 [1, 2]. Quiescent FRS2 is membrane anchored through its $\mathrm{N}$-terminal myristoylation site and is constitutively bound to FGFR through its PTB domain. Upon FGF stimulation, the lipid-anchored FRS2 rapidly undergoes tyrosine phosphorylation (PY), generating at least six functional pTyr-binding sites. FRS2 then forms

Correspondence: Zhengjun Chen

Tel: +86-21-54921081; Fax: +86-21-54921081

E-mail: zjchen@sibs.ac.cn

Received 11 August 2008; revised 25 March 2009; accepted 27 April 2009; published online 4 August 2009 a complex with the tyrosine phosphatase Shp2 and the adapter protein Grb2, which associates with the Ras activator Sos. The FRS2-Grb2-Sos ternary complex then activates the Ras/mitogen-activated protein kinase (MAPK) signaling cascade $[1,3-6]$. PY of FRS2 is also reported to play an important role in PI3 kinase activation through the formation of the FRS2-Grb2-Gab1 complex. FRS2induced activitation of PI3 kinase results in regulated cell migration [7]. Hence, PY of FRS2 is considered an early marker of FGF pathway activation. In addition, FRS2 has been reported to undergo PY in FGFR, NGFR, PDGFR, BDNFR, and VEGFR signaling pathways [2, 8-11], demonstrating its wide role in signal transduction.

As the major signal transducer in the FGFR pathway, FRS2 can potentially integrate positive as well as negative signal inputs. Negative feedback can be generated through the formation of a complex among PY-FRS2, Sprouty, and the E3 ubiquitin ligase $\mathrm{Cbl}$, resulting in ubiquitination and degradation of both FRS2 and FGFR1 
$[12,13]$. In addition, activated MAPKs can phosphorylate FRS2 on several threonine residues, leading to reduced PY of FRS2. Reduced PY results in decreased recruitment of Grb2, reduced MAPK activity, and consequent attenuation of FGF signal transduction [14, 15]. MAPKs are key components in a variety of signaling pathways that respond to cellular stimuli such as growth factors, cytokines, and GPCR ligands [16-18]. Moreover, threonine phosphorylation (PT) of FRS2 was observed upon stimulation of several ligands $[14,15]$. Thus, the FGF pathway is likely to sense and crosstalk with other signaling pathways through FRS2.

Crosstalk between signaling pathways allows intracellular networks to produce diverse cellular responses [1922]. However, it is quite difficult to study the molecular mechanisms of crosstalk among growth factor signal transduction pathways because many growth factor pathways make use of the same downstream signaling machinery. This characteristic makes it difficult to choose an entry point to study crosstalk. In this work, we investigated whether FRS2 is a substrate for signaling pathway crosstalk. Indeed, FGF-induced PY of FRS2 could be considerably reduced by EGF. This effect was reversed by the MEK1 inhibitor U0126, indicating that the ERK2FRS2 negative regulatory loop may receive signals from the EGF pathway. We also generated a site-directed mutant, FRS2-3KL, which cannot bind to ERK2 or undergo $\mathrm{PT}$ in response to EGF or FGF stimulation. This mutant was resistant to EGF-induced PT and produced enhanced PC12 cell neuronal differentiation. Our data suggest that PT of FRS2 may function as a molecular switch in the FGF pathway, sensing and participating in crosstalk with other signaling pathways.

\section{Results}

EGF co-stimulation attenuates FGF-induced PY of FRS2 in an ERK1/2-dependent manner

FRS2 undergoes PY as well as PS/T. The pThr motif (pTP), a conserved phosphorylation motif in ERK2 substrates, was identified as a phosphorylated threonine followed by a proline [14, 23, 24]. Using the 293T/FGFR1 cell line, we investigated the phosphorylation status of ectopic FRS2 in response to extracellular stimulation with different compounds by using antibodies specifically against pTP or pY. Besides growth factors, GPCR ligands are also strong ERK agonists and thus may induce the phosphorylation of FRS2. Stimulation with EGF, as well as with the GPCR ligands thrombin and lysophosphatidic acid (LPA), induced PT of FRS2 in the absence of PY, while the phosphatase pan-inhibitor POV provoked both PT and PY of FRS2 (Figure 1A).
We observed similar results when insulin and insulinlike growth factor-1, but not PDGF, were applied to DAL cells, a human mammary carcinoma cell line. Here, PT of FRS2 was indicated by an apparent molecular weight upshift (Supplementary information, Figure S1A). Coimmunoprecipitation of FRS2 with MAPK/ERK2 was detected in response to all types of stimuli (Figure 1A), underscoring the role of ERK2 in PT of FRS2.

The phosphorylation status of endogenous FRS2 was also characterized after stimulation of PC12 cells with aFGF in the presence or absence of EGF. We observed that EGF/FGF co-stimulation considerably reduced the FGF-induced PY of FRS2 to approximately 50\% (Figure 1B). This phenomenon could be reversed by adding the MEK1 inhibitor U0126 (Figure 1B), indicating the involvement of the ERK2-FRS2 regulatory loop. Similar results were obtained in NIH3T3 and Ovcar-3 cells (Supplementary information, Figure S1B).

Co-immunoprecipitation was performed to determine if the attenuation of PY affects the recruitment of downstream molecules by FRS2. PC12 cells were transfected with pRK5-flag-FRS2, followed by immunoprecipitation with anti-flag antibody. FGF stimulation greatly elevated the recruitment of endogenous Grb2; however, co-stimulation with EGF inhibited the FRS2-Grb2 interaction. This inhibition occurred in parallel to the suppression of PY of FRS2 (Figure 1C). With this 10-min treatment, EGF co-stimulation did not inhibit activation of ERKs or Akt, indicating the importance of using the appropriate time window to observe the outcomes of EGF/FGF costimulation (Figure 1C).

\section{Identification of the ERK2-docking site in FRS2}

To further study the molecular mechanism underlying EGF-induced inhibition of FRS2 PY, we constructed a series of FRS2 deletion mutants. Using pull-down assays, the affinity of these FRS2 mutants for ERK2 was examined (Figure 2A and Supplemental information, Figure S2). We found that the aa 233-272 region of FRS2 was critical for ERK2 docking, as a GST fusion protein comprising only this short peptide ( $\Delta 2 \mathrm{com})$ was sufficient to pull down nearly the same amount of ERK2 as the fulllength FRS2. Half-CT, a fusion protein composed of aa 248-508, can also bind ERK2, while Half-NT, which is composed of aa 1-247, does not exhibit any affinity for ERK2 (Figure 2A). Thus, the aa 248-272 stretch of FRS2 should contain the binding region for ERK2.

Site-directed mutagenesis was performed to determine the residues important for ERK2 binding. Three mutants were constructed: FRS2 point mutant A (FPA), wherein the region $\mathrm{K}^{250} \mathrm{FVL}^{253} \mathrm{GPTPVQK}^{260} \mathrm{QL}^{262}$ was mutated to $\mathrm{N}^{250} \mathrm{FVF}^{253} \mathrm{GPTPVQN}^{260} \mathrm{QF}^{262}$; FRS2 point mutant $\mathrm{B}$ 

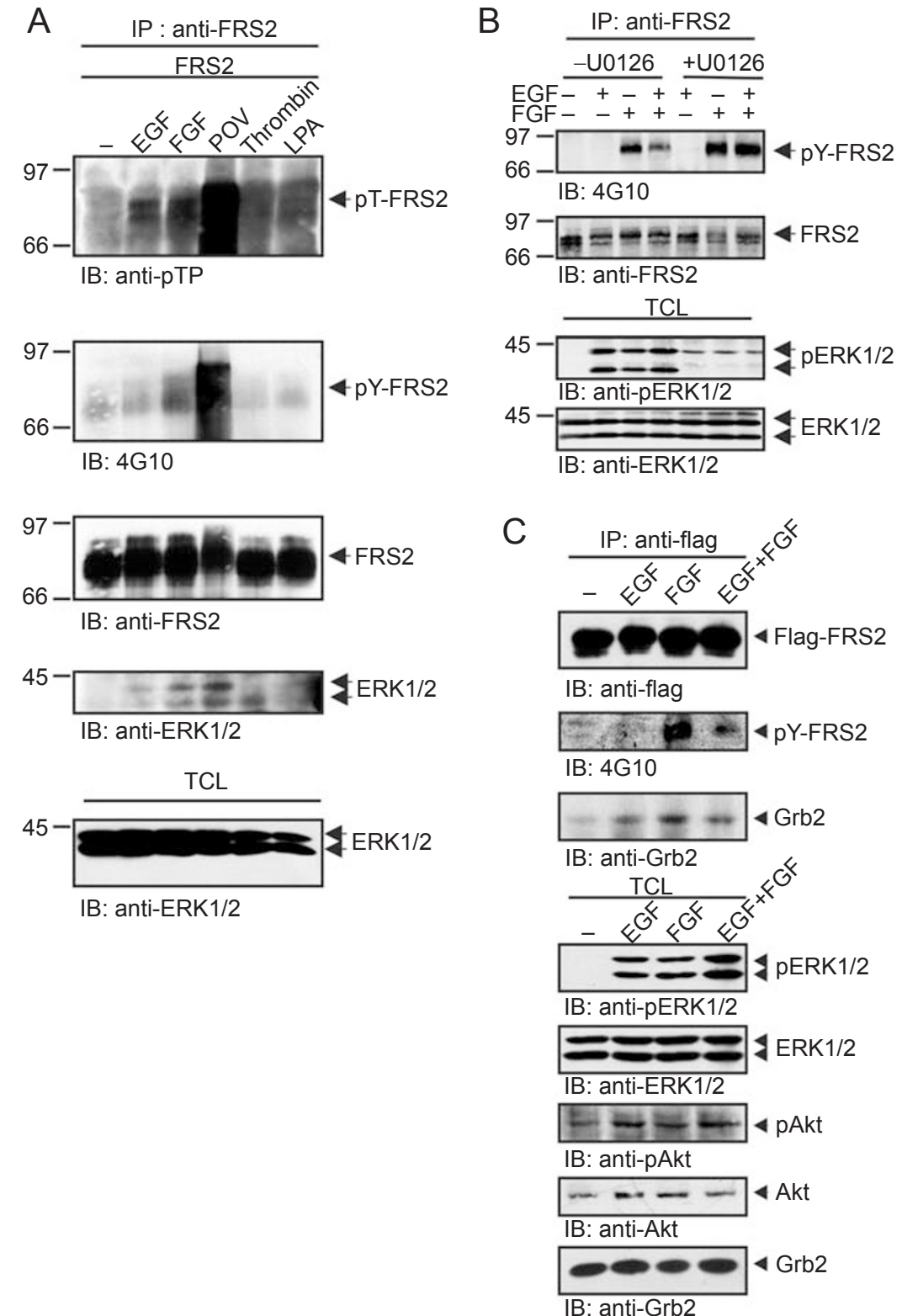

Figure 1 MAPK-induced Ser/Thr phosphorylation inhibits PY of FRS2 in PC12 cells. (A) Multiple stimulations provoked PT of FRS2. FRS2 was transfected into 293T/FGFR1 cells, followed by stimulation with EGF (100 ng/ml), FGF (20 ng/ml), POV (1 mM), thrombin $(1 \mathrm{U})$, or LPA $(5 \mathrm{mM})$ for $10 \mathrm{~min}$. FRS2 phosphorylation was monitored by anti-pTP or $4 \mathrm{G} 10$ antibody. (B) EGF co-stimulation substantially attenuates FGF-induced PY of FRS2 in an ERK2-dependent manner. PC12 cells $(2 \times 100$ $\mathrm{mm}$ dishes) were stimulated by FGF $(20 \mathrm{ng} / \mathrm{ml})$ in the presence or absence of EGF $(100 \mathrm{ng} / \mathrm{ml})$ as well as the MEK1 inhibitor, U0126 (5 mM). (TCL, total cell lysates) (C) EGF co-stimulation inhibits FGF-induced FRS2-Grb2 interaction. PC12 cells were transfected with Flag-FRS2, serum-starved overnight, and then stimulated with FGF, EGF or both. Cell lysates were subjected to immunoprecipitation with anti-flag antibody, and co-immunoprecipitated Grb2 was monitored with anti-Grb2 antibody. pERKs, pAkt, total ERKs and Akt were monitored with corresponding antibodies.

(FPB), wherein the region $\mathrm{K}^{260} \mathrm{QL}^{262} \mathrm{MEKEK}^{267} \mathrm{~L}^{268}$ was mutated to $\mathrm{N}^{260} \mathrm{QF}^{262} \mathrm{MEKEN}^{267} \mathrm{~V}^{268}$; and FRS2-3KL, wherein $\mathrm{K}^{250} \mathrm{FVL}^{253} \mathrm{GPTPVQK}^{260} \mathrm{QL}^{262} \mathrm{MEKEK}^{267} \mathrm{~L}^{268}$ was mutated to $\mathrm{N}^{250} \mathrm{FVF}^{253}$ GPTPVQN ${ }^{260} \mathrm{QF}^{262}$ MEK$\mathrm{EN}^{267} \mathrm{~V}^{268}$ (Figure 2B). The results of GST pull-down assays revealed that FPA significantly lost affinity for ERK2, whereas formation of an FPB-ERK2 complex was only slightly reduced (Figure 2C). Strikingly, the complex was completely abolished when FRS2-3KL was used as the bait (Figure 2C). These data suggest that the 
A

\begin{tabular}{|c|c|c|c|c|c|}
\hline Names & & & Struc & & \\
\hline & & & 152 & & 508 \\
\hline WT & GST [ & PTB & 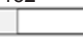 & & \\
\hline & & & $152 \quad 247$ & & \\
\hline Half-NT & GST [ & PTB & $\square$ & & \\
\hline & CST & & 248 & & 508 \\
\hline tall-c। & GSI & & & 326 & 508 \\
\hline CT & GST & & & & \\
\hline & & & 238 & 34 & \\
\hline $\mathrm{CP}$ & GST & & & 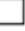 & \\
\hline & 1 & & 152231 & & 508 \\
\hline$\Delta 1$ & GST & PTB & ב & & 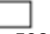 \\
\hline 2 & GST & PTR & 152231 & & 50 \\
\hline & - & TID & & & \\
\hline zcom & GST & & \begin{tabular}{|l} 
\\
\end{tabular} & & \\
\hline & COT & & 152260 & & 508 \\
\hline & GST & PTB & 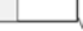 & & \\
\hline & GST & & 152250 & & 508 \\
\hline
\end{tabular}

B

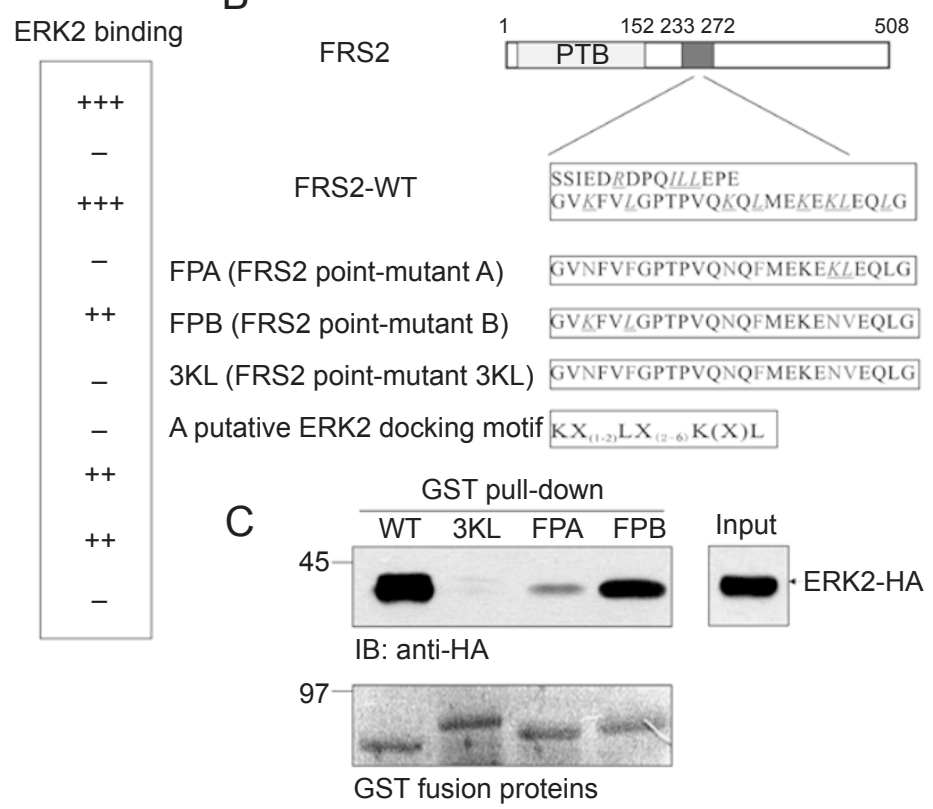

Figure 2 Identification of the ERK2-binding motif in FRS2. (A) Schematics of GST-FRS2 fusion proteins and their affinity for ERK2. (B) A putative novel ERK2-docking motif in FRS2. A narrow 40-amino-acid region that showed full affinity for ERK2 was discovered. Positively charged lysine residues and hydrophobic leucine residues, the most important components of classical ERK2 conserved docking domains, are in italics and underlined. A putative novel ERK2-docking motif was proposed, and three site-directed FRS2 mutants were generated accordingly. (C) A point mutant FRS2-3KL displays complete loss of affinity for ERK2. The amount of the loaded GST fusion proteins was quantified by amido black staining.

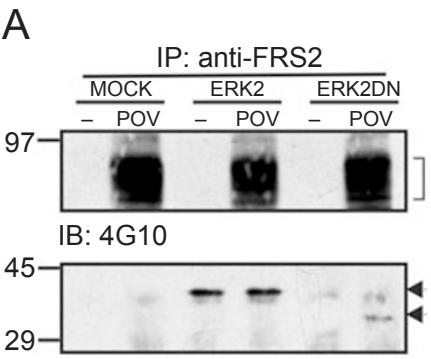

IB: anti-ERK1/2

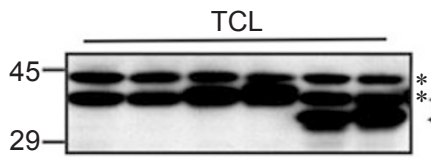

IB: anti-ERK1/2
B

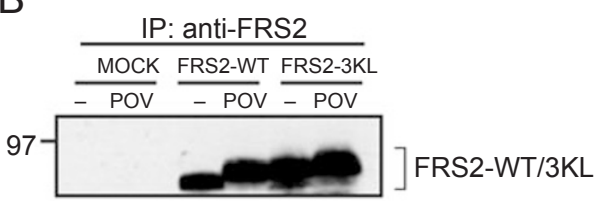

IB: anti-FRS2

ERK2 ERK2DN

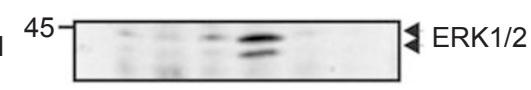

IB: anti-ERK1/2

TCL

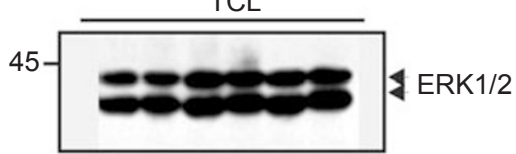

IB: anti-ERK1/2

Figure 3 ERK2 activity-dependent interaction between FRS2 and ERK2. (A) The CD domain in ERK2 is responsible for the FRS2-ERK2 interaction. ERK2-HA or ERK2DN-HA was transfected into 293T cells. Cells were stimulated with 1 mM POV or kept quiescent. Co-immunoprecipitation was performed with anti-FRS2 antibodies. The point mutant ERK2DN largely lost its affinity for FRS2. Activated endogenous FRS2 in 293T cells was detected using 4G10 antibody. Co-immunoprecipitated ERK2 was detected by western blotting using anti-ERK1/2. (*, endougenous ERK1/2) (B) Wild-type FRS2 but not FRS2-3KL binds to endogenous ERK2 in 293T cells upon POV stimulation. FRS2 or FRS2-3KL was transfected into 293T cells. Cells were stimulated with $1 \mathrm{mM}$ POV or kept quiescent. Co-immunoprecipitation was performed with anti-FRS2 antibodies. Coimmunoprecipitated endogenous ERKs were detected with anti-ERK1/2 antibodies. 
$\mathrm{K}^{250} \mathrm{FVL}^{253} \mathrm{GPTPVQK}^{260} \mathrm{QL}^{262}$ region in FRS2 should be the major ERK2 docking site.

The existence of a conserved docking domain, that is, $\mathrm{K} / \mathrm{RK} / \mathrm{RK} / \mathrm{R} \ldots \mathrm{I} / \mathrm{LXI} / \mathrm{L}$ [25-27], is a shared feature of the activators, effectors, and substrates of ERK2. Although the same sequence was not detected in the aa 248-272 region, the most important components of this domain the positively charged lysine residues and the hydrophobic leucine residues - were spread throughout the region. In combination with the results of our pull-down assays using the point-mutated FRS2, our data suggest that a putative novel ERK2-docking motif, namely, $\mathrm{KX}_{(1-2)}$ $\mathrm{LX}_{(2-6)} \mathrm{K}(\mathrm{X}) \mathrm{L}$, is essential for the formation of the FRS2ERK2 complex (Figure 2B).

\section{Identification of the FRS2-docking site in ERK2}

It has been reported that the Common Docking (CD) domain in ERK serves as a general site for the recognition of its activators, substrates, and regulators [26]. Therefore, we constructed ERK2DN, a point mutant of mouse ERK2, in which the key residues in CD domain, Asp316 and Asp319, are mutated to glycines. ERK2-HA or ERK2DN-HA was overexpressed in 293T cells, followed by immunoprecipitation with anti-FRS2 antibody.
Although a large amount of WT ERK2 co-immunoprecipitated with FRS2, only a trace quantity of ERK2DN could be detected (Figure 3A). Thus, the CD domain of ERK2 might serve as the docking site for FRS2. To further analyze the interaction between FRS2 and ERK2, co-immunoprecipitation was performed against endogenous ERK2. FRS2 or FRS2-3KL was transfected into HEK293T cells. Endogenous ERK1/2 could only be coimmunoprecipitated with FRS2-WT but not FRS2-3KL. Furthermore, this interaction could only be detected in POV-stimulated cells but not in quiescent cells (Figure 3B). However, overexpressed ERK2 could associate with FRS2 in the GST pull-down assays as well as in the co-immunoprecipitation experiments (Figures 2C and $3 \mathrm{~A})$. Based on these observations, it appears that overexpressed ERK2 can constitutively bind to FRS2, while the interaction between endogenous ERK2 and FRS2 is substantially elevated after POV-induced ERK1/2 activation. Thus, we suggest that endogenous ERK2 may be recruited by FRS2; however, this process is probably prevented by cellular compartmentalization and can occur only after extracellular stimulation. However, ERK2 overexpression may lead to dispersed distribution and constitutive interaction between ERK2 and FRS2.

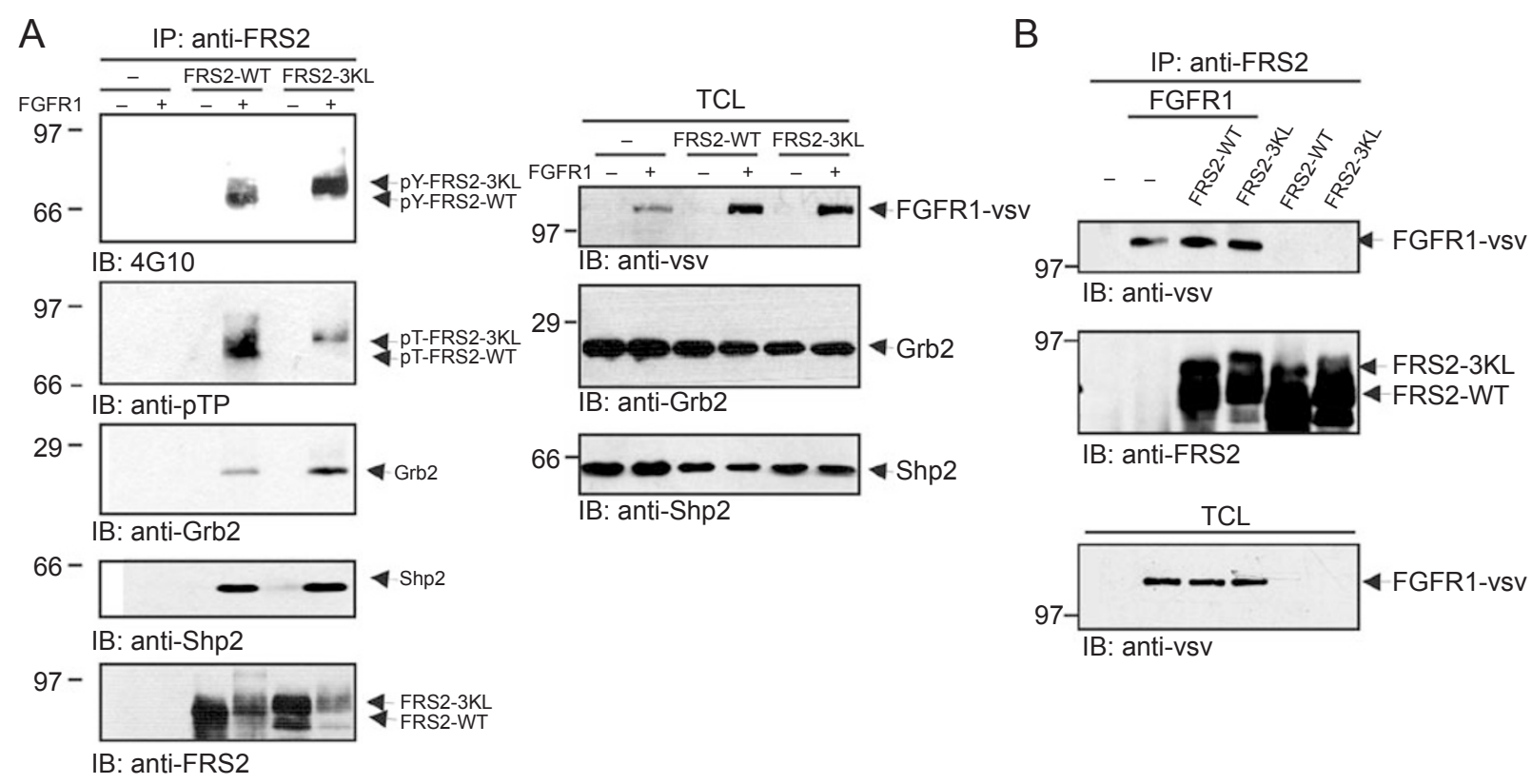

Figure 4 The point mutant FRS2-3KL enhances FGFR1-mediated cell signaling. (A) Disruption of the ERK2-docking motif increases PY of FRS2 and the subsequent recruitment of downstream signal molecules in response to FGFR1 activation. FRS2 or FRS2-3KL was transfected into 293T cells with 50 ng FGFR1 or pRK5 empty vector. The products of co-immunoprecipitation were western blotted with anti-pTP, anti-Shp2, anti-Grb2, or $4 \mathrm{G} 10$ antibodies. Total cell lysate blotted with different antibodies were presented as the control for input. (B) Disruption of the ERK2-docking motif in FRS2 does not affect its affinity for FGFR1. FRS2 or FRS2-3KL was transfected into 293T cells with FGFR1-vsv or empty vector. FGFR1 bound to FRS2WT or FRS2-3KL was detected using the anti-vsv antibody. 
Disruption of ERK2 docking increases PY of FRS2 and enhances downstream signal transduction of the FGF signaling pathway

The PT of FRS2 induced by the FRS2-ERK2 negative feedback loop was reported to inhibit PY of FRS2 in response to FGF stimulation as well as, theoretically, the downstream signal transduction of the FGF signaling pathway. It is difficult to directly prove that PT of FRS2 induces inhibition of the FGF signaling pathway. However, abolishment of ERK2 docking and PT of FRS2 should enhance PY of FRS2 and the subsequent signal transduction events. FRS2-WT or FRS2-3KL was cotransfected into 293T cells along with FGFR1. After immunoprecipitation with anti-FRS2 antibody, the samples were subjected to SDS-PAGE followed by western blotting. As shown in Figure 4A, FRS2-WT was strongly tyrosine phosphorylated as well as threonine phosphorylated when coexpressed with FGFR1. In contrast, while PY of FRS2-3KL was stronger than that of FRS2WT, only a trace amount of FRS2 PT was observed for this mutant (Figure 4A). As a consequence of enhanced PY, FRS2-3KL recruited more Grb2 than did FRS2-WT (Figure 4A). However, the recruitment of Shp2 by FRS2$3 \mathrm{KL}$ seemed only slightly enhanced, probably due to saturation of the signal (note that even in the absence of FGFR1, there was detectable Shp2 binding to the mutant). Thus, elimination of associated ERK2 enhanced the capacity of FRS2 to relay signals from FGFR1; that is, PT of FRS2 inhibited subsequent signal transduction in the FGF signaling pathway. Notably, inhibition of FRS2 PT did not affect its affinity for FGFR1 (Figure 4B).

Disruption of ERK2 docking abolishes PT of FRS2 and EGF-induced attenuation of PY of FRS2

Subsequently, we examined the phosphorylation status of the FRS2-3KL mutant upon EGF stimulation. Stimulation with $100 \mathrm{ng} / \mathrm{ml}$ EGF for $3 \mathrm{~min}$ produced strong PT of FRS2-WT, which persisted for more than 90 min. In contrast, PT of FRS2-3KL could barely be visualized in the total cell lysate (Figure 5A). Although traces of threonine-phosphorylated FRS2-3KL appeared in the immunoprecipitates after a 3-min stimulation, it disappeared soon after (Figure 5A). Since we could only detect co-immunoprecipitated ERK2 in the presence of FRS2-WT but not the mutant, we ascribed the weak PT of FRS2-3KL to its indirect interaction with ERK2 or other kinases.

To reconstitute the EGF co-stimulation-induced attenuation of FRS2 PY, FRS2-WT or FRS2-3KL was transfected into 293T/FGFR1 stable-transfected cells followed by FGF treatment in the presence or absence of EGF. As expected, EGF co-stimulation greatly reduced
PY of FRS2-WT but not FRS2-3KL in response to FGF stimulation (Figure 5B). These data show that EGFactivated ERK2 can bind to FRS2 and cause PT, which in turn suppresses PY induced by FGF stimulation.
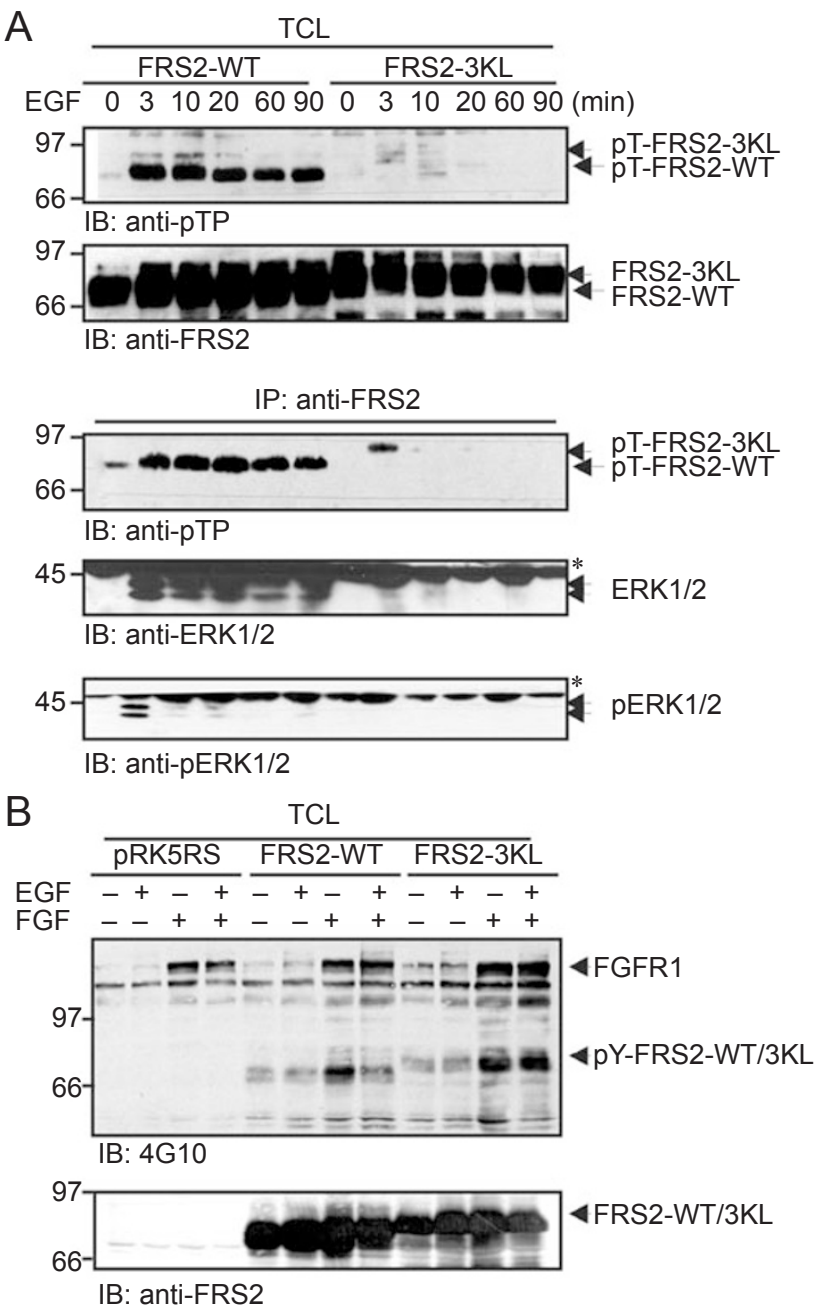

Figure 5 EGF-induced suppression of FRS2 PY upon FGF stimulation does not occur with the point mutant FRS2-3KL. (A) Disruption of ERK2 docking abolishes PT of FRS2. FRS2 or FRS2-3KL was transfected into 293T cells. Serum-starved cells were subjected to EGF time course stimulation. Cells were lysed and incubated with anti-FRS2. Immunoprecipitated proteins were detected by western blotting using anti-pTP for pTFRS2, anti-ERK1/2 for co-immunoprecipitated total ERK, and anti-pERK1/2 for activated ERKs. (*, heavy chain) Total cell lysates are shown as input controls. (B) Disruption of ERK2 docking abolishes EGF-induced suppression of PY of FRS2 in response to FGF stimulation. FRS2 or FRS2-3KL was transfected into 293T/FGFR1 stable-transfected cells. Serum-starved cells were stimulated with FGF, EGF or both. PY of FGFR1 and FRS2 were monitored with 4G10. The expression of wild-type FRS2 and FRS2-3KL were detected using anti-FRS2. 
A

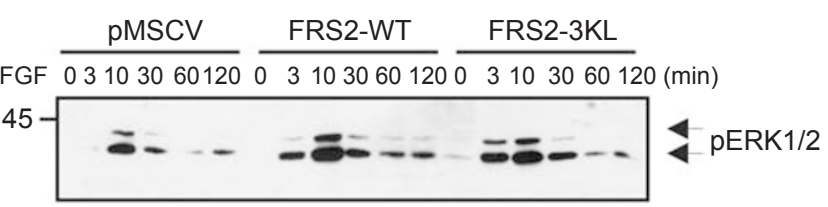

IB: anti-pERK1/2
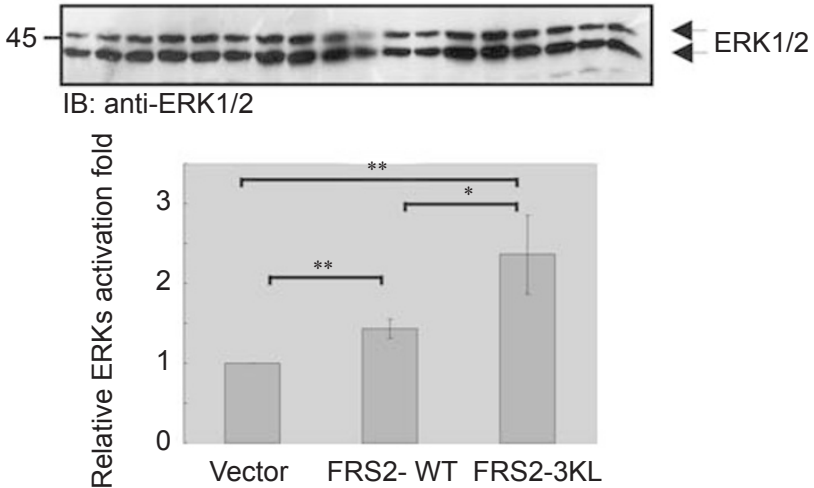

ERK2 activity at 3 min after FGF stimulation

B
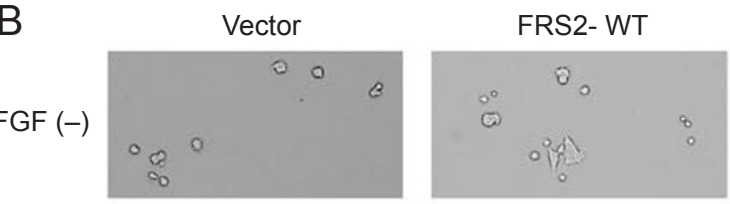

FRS2-3KL
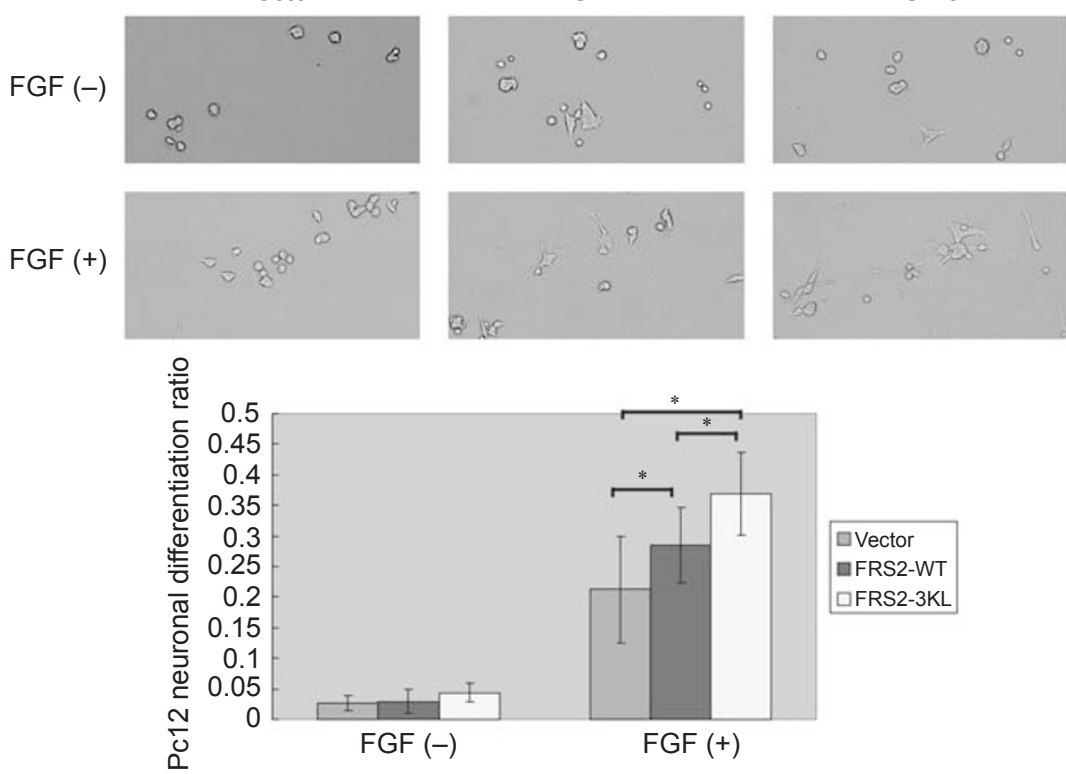

Figure 6 Different effects on ERK1/2 activation and PC12 differentiation by FRS2-WT and FRS2-3KL. (A) FRS2-WT and FRS2-3KL induce different kinetics of ERK2 activation in 3T3 cells upon aFGF stimulation. 3T3 cells transfected with pMSCV vector, FRS2-WT, or FRS2-3KL were stimulated with $50 \mathrm{ng} / \mathrm{ml}$ aFGF for different durations after serum-starvation. Three repetitive experiments were performed, and the ERK1/2 activation status 3 min after aFGF stimulation was quantified by QuantiteOne (Bio-Rad) and statistically analyzed. (**P<0.01, $* P<0.05)$ (B) PC12 neuronal differentiation assays were carried out as described in Materials and Methods. pMSCV vector, FRS2-WT, or FRS2-3KL were transfected into the PC12 cells. PC12 morphology was analyzed under a microscope. After $48 \mathrm{~h}$, neurite outgrowth was scored and quantified. Cells exhibiting a dendrite length twice that of the cell body were considered differentiated. Three repetitive experiments were performed, and the obtained data were statistically analyzed. $(* P<0.05)$.

FRS2-WT and FRS2-3KL induce differential ERK2 activation in response to FGF stimulation

ERK2 is one of the key components of the FGF signaling pathway, which is involved in cell proliferation, differentiation, and other important cellular responses. Therefore, the activity kinetics of this enzyme can be regarded as a potential marker of FGF signaling pathway activation. According to previous reports, mutation 
A

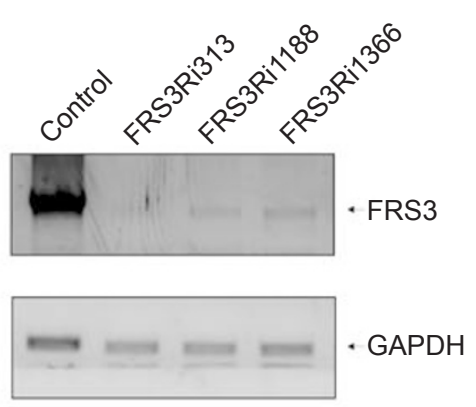

B

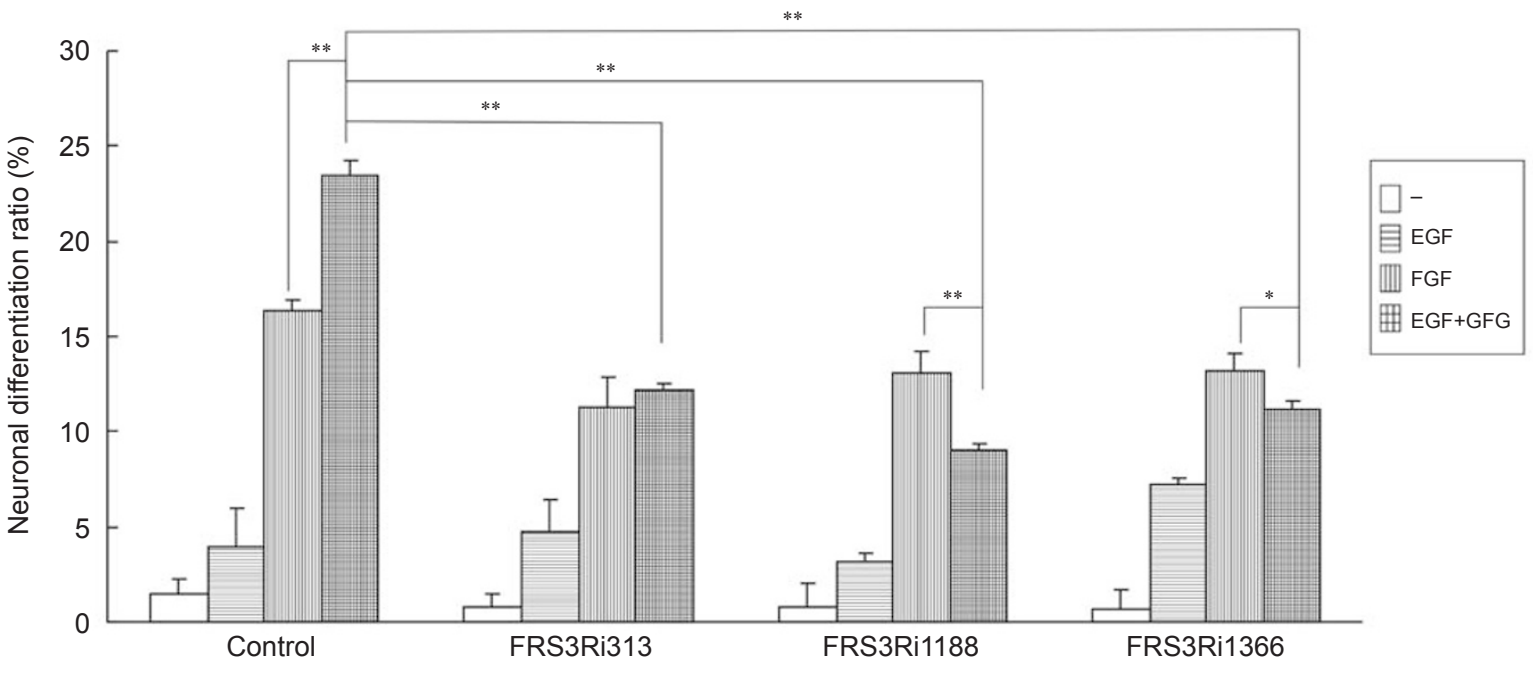

C

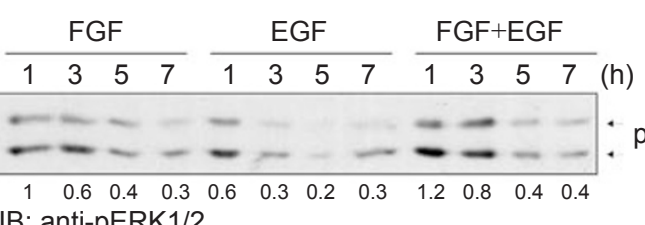

pERK1/2

IB: anti-pERK1/2

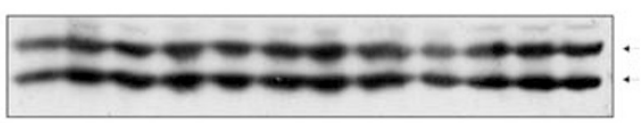

IB: anti-ERK1/2
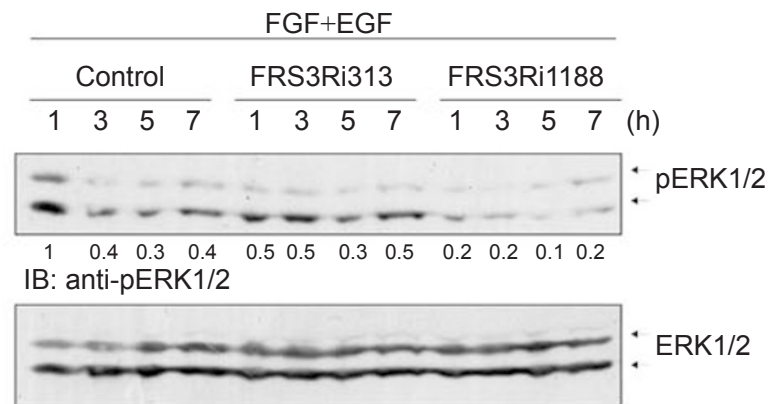

ERK1/2

Figure 7 FRS2 may mediate the negative effect of EGF on the FGF pathway in PC12 cells. (A) Efficiencies of siRNAs for FRS3. PC12 cells were transfected with NC or siRNAs, and 3 days later, total mRNA was extracted. Reverse transcription was performed, and the cDNA products were used as templates for PCR to determine the mRNA levels of endogenous FRS3. GAPDH mRNA levels were used as an internal standard. (B) PC12 neuronal differentiation assays were carried out as described in Materials and Methods. Control, FRS3Ri313, FRS3Ri1188 or FRS3Ri1366 was transfected into PC12 cells. PC12 morphology was analyzed under a microscope. After $48 \mathrm{~h}$, neurite outgrowth was scored and quantified. Cells exhibiting a dendrite length twice that of the cell body were considered differentiated. Three repetitive experiments were performed, and the obtained data were statistically analyzed. (t-test $* P<0.05 ; * * P<0.01$ ) (C) PC12 cells transfected with different siRNAs were serum-starved and stimulated with EGF, FGF or EGF plus FGF for indicated durations. The activities of ERK1/2 were monitored by anti-pERK1/2 antibodies.

of eight threonine residues in FRS2 leads to enhanced ERK2 activity [14]. Therefore, we hypothesized that expression of the FRS2-3KL mutant, which cannot undergo
PT, should also result in greater ERK2 activity.

To investigate the effects of FRS2-3KL on FGF signaling, $3 \mathrm{~T} 3$ cells were infected with recombinant retrovirus 
carrying an empty pMSCV vector, pMSCV-FRS2-WT, or pMSCV-FRS2-3KL. At $24 \mathrm{~h}$ post infection, the cells were serum-starved overnight and then stimulated with $50 \mathrm{ng} / \mathrm{ml}$ aFGF. Our results showed that while FRS2WT greatly elevated the ERK2 activity compared to the control, FRS2-3KL raised the ERK2 activity to an even higher level within a specific time window (less than 10 min; Figure 6A). However, FRS2-3KL did not alter the duration of the ERK2 activity compared to FRS2-WT. These results indicate that disruption of the FRS2-ERK2 interaction enhances the activity of the FRS2-mediated FGF signaling pathway.

\section{Influence on PC12 differentiation by FRS2-3KL}

The rat pheochromocytoma cell line PC12 is a welldocumented model for studying cellular responses to growth factor stimulation. To study the biological relevance of the FRS2-3KL mutant, PC12 neuronal differentiation assays were carried out. As previously reported, overexpressed FRS2-WT enhances FGF signaling and thereby provokes neuronal differentiation of PC12 cells. Differentiated cells can be identified by the presence of flattened cell bodies and neurite outgrowths (Figure 6B). PC12 cells infected with FRS2-3KL showed the highest differentiation ratio in response to FGF stimulation (Figure $6 \mathrm{C}$ ), which is possibly due to the elimination of the FRS2-ERK2 negative feedback loop resulting from the abolishment of ERK2 docking.

FRS2 may mediate the negative effect of EGF on the $F G F$ pathway in $P C 12$ cell differentiation

When PC12 cells were stimulated with EGF/FGF, more cells underwent neuronal differentiation compared to stimulation with FGF alone (data not shown). This result conflicts with the observation that EGF/FGF stimulation attenuates PY of FRS2 compared with FGF alone. Given that the homolog FRS2 $3 /$ FRS3 was heavily tyrosine phosphorylated upon FGF stimulation, and that this phosphorylation would not be inhibited by EGF co-stimulation, FRS $2 \beta /$ FRS 3 may compensate for the FRS2-mediated inhibitory effects of EGF on the FGF pathway. Thus, we designed three siRNAs (FRS3Ri313, FRS3Ri1188, and FRS3Ri1366) to reduce FRS23/FRS3 levels and then observed the effect of EGF/FGF co-stimulation on neuronal differentiation. As shown in Figure 7A, all three siRNAs could efficiently knock down endogenous FRS2 $\beta / F R S 3$. Neuronal differentiation assays demonstrated that knockdown of FRS3 suppressed FGFinduced neuronal differentiation to some extent (Figure 7B). In control-transfected cells, EGF co-stimulation elevated FGF-induced neurite growth, whereas in cells transfected with FRS3Ri1188 or FRS3Ri1366, EGF co- stimulation decreased the FGF-induced neuronal differentiation of the cells (Figure 7B). For unknown reasons, this phenomenon was not observed in PC12 cells transfected with FRS3Ri313. However, on the whole, knockdown of FRS3 in PC12 cells uncovered the negative effect of EGF on the FGF pathway. In addition, we observed that all three RNAi constructs led to dramatic suppression of neuronal differentiation in response to EGF/FGF co-stimulation compared with control, indicating an important role of FRS3 in mediating the signals from EGF/FGF co-stimulation (Figure 7B). We also examined the activity of ERKs in these cells. FGF stimulation of control-transfected cells resulted in sustained activation of ERKs, while EGF stimulation led to relatively transient activation of ERKs. We found that EGF/FGF co-stimulation produced even greater activation of ERKs without reduction in duration. However, in cells transfected with FRS3Ri1188, activation of ERKs in response to EGF/FGF co-stimulation dramatically decreased as compared with control-transfected cells (Figure 7C).

\section{Discussion}

Growth factors such as FGF and EGF play important roles in controlling various aspects of morphogenesis, migration, proliferation, and differentiation. Growth factors form a complex cellular signaling language, in which combinations of individual growth factors can produce diverse effects. Therefore, crosstalk between different signaling pathways is likely to occur. In this report, we examined whether the adapter protein FRS2 is a potential substrate of crosstalk between the FGF pathway and other signaling pathways. As described previously, FRS2 undergoes strong PS/T in response to several extracellular stimuli $[1,15,28]$; however, a plausible explanation for the function of this phosphorylation was not reported. Recently, a negative feedback loop comprising FRS2 and MAPK/ERK has been described. In this model, FGF stimulation leads to PT of FRS2 by MAPK/ERK, resulting in decreased PY of FRS2 and reduced downstream FGF signaling $[14,15]$. Consequently, we sought to determine whether the FGF pathway could sense other stimuli via FRS2. We found that FRS2 can receive external regulatory signals from the EGF pathway and can be regulated via MAPK-induced PS/T. In this way, the FGF pathway might participate in the crosstalk with the EGF pathway as well as with other pathways.

We found that a region of aa 248-272 in FRS2 $\alpha$ contains the binding site for ERK2, which is consistent with the results of a previous study reporting the aa 237-252 stretch as the FRS2-ERKs binding region in FRS2 $\beta$ [29, 30]. The most important components of this region are 
the positively charged lysine residues and the hydrophobic leucine residues. The CD domain, that is, DXXD, comprising two negatively charged aspartic acids and conserved in rat, mouse, and human ERK2 [26, 31], may potentially interact with the positively charged lysine residues in the docking region of FRS2 (Figure 2B). It has been reported that activity-dependent exposure of the $\mathrm{CD}$ domain is very important for the interaction between ERK2 and its partners [32]. Consistent with this result, our experiments showed that endogenous ERK2 could only be co-immunoprecipitated with FRS2 after stimulation, independent of PY of FRS2 (Figures 5A and 3B). Therefore, we suggest that ERK2 docking on FRS2 depends on ERK2 activity but not on the PY of FRS2, indicating that any stimuli capable of activating ERK2 may lead to the formation of the FRS2-ERK2 complex and the subsequent PT of FRS2.

The homolog FRS2 $\beta / F R S 3$, which shares high similarity with FRS2 $\alpha$, was reported to interact with ERK1/2 and inhibit ERK1/2 activation in the EGF pathway [29, 30]. This inhibitory effect did not occur with FRS2 $\alpha$ (Supplementary information, Figure S3). Lax et al. [14] reported that FRS2 $\beta$ lacks an MAPK phosphorylation motif and cannot be threonine-phosphorylated. It does not undergo an electrophoretic mobility shift in response to FGF stimulation. Thus, we hypothesized that EGF could not negatively regulate FRS $2 \beta$ PY induced by FGF. Our data supported this notion (Supplementary information, Figure S1D). Since FRS2 $\beta$ is specifically expressed in neuronal tissues, while FRS2 $\alpha$ is widely expressed in several kinds of tissues, these two homologs may have quite different functions.

In addition, it is unclear why PT of FRS2 would inhibit PY. IRS-1 is the counterpart of FRS2 in the insulin signaling pathway and exhibits balanced PY and PT. Upon PS/T, either IRS-1 becomes a poor substrate for insulin receptor kinase [33] or its association with subcellular compartments and/or stability is probably altered [34, 35]. FRS2 may also undergo similar changes. According to our data (Figure 4B), PT did not produce a conformational change that prevents the interaction between FGFR1 and FRS2, a mechanism suggested previously [14].

To study the role of the ERK2-FRS2 negative feedback loop and the subsequent PT of FRS2 in EGF and FGF pathway crosstalk, we generated a mutant FRS2$3 \mathrm{KL}$, wherein the putative ERK2-docking domain was mutated. Although we could not elucidate whether other residues besides the six amino acids mutated in the aa 248-272 region are dispensable for the ERK2-FRS2 interaction, neither interaction with ERK2 nor obvious PT was observed in FRS2-3KL after EGF or FGF stimula- tion. The mutated FRS2 exhibited affinity for its downstream targets and was able to activate ERK2, suggesting that these point mutations did not lead to a structural collapse of FRS2. As expected, FGF-induced PY of FRS2$3 \mathrm{KL}$ could not be attenuated by EGF co-stimulation, and the mutant displayed increased recruitment of downstream signaling molecules in response to FGFR1 activation. In addition, cells expressing the mutant FRS2 exhibited elevated activation of ERKs as well as a higher PC12 neuronal differentiation ratio upon FGF stimulation compared to cells expressing FRS2-WT. In 293T/ FGFR1 cells, in which Akt was in a constitutively active state as a result of stably overexpressed FGFR1, transfection of FRS2-WT inhibited Akt activity in response to EGF stimulation, while transfection of mutant FRS2$3 \mathrm{KL}$ showed no effect on Akt activation (Supplementary information, Figure S5). These data suggest that FRS2 can tune the FGF signaling pathway via ERK-mediated PT.

To investigate whether signals from other signaling pathways can influence the FGF pathway via FRS2, we used PC12 cells $[22,36]$ to study the effect of EGF costimulation on cell differentiation. Generally, EGF is believed to induce proliferation, while FGF or NGF is believed to provoke neuronal differentiation of PC12 cells [22]. Strikingly, when PC12 cells were treated with EGF in the presence of FGF for $48 \mathrm{~h}$, neuronal differentiation was enhanced, not inhibited, compared to stimulation with FGF alone (data not shown). According to our experiments, the homolog FRS2 $3 / F R S 3$ would be heavily tyrosine phosphorylated upon FGF stimulation, and this phosphorylation would not be inhibited by EGF co-stimulation. Therefore, it is likely that FRS2 $\beta / F R S 3$ plays an important role in PC12 differentiation. We then generated RNAi constructs against FRS2 $\beta / F R S 3$ and found that EGF co-stimulation significantly inhibited FGF-induced neuronal differentiation in cells depleted of FRS2 $\beta /$ FRS3, but not in negative control-transfected cells. It has been reported that differentiation activity occurs in parallel with phosphorylation of FRS2 and ERK1/2 in response to FGF [1, 37, 38]. Our data suggest that inhibition of ERK1/2 activity over a long duration may underlie the inhibitory effect of EGF on FGFinduced PC12 differentiation in FRS2 $\beta$ /FRS3-depleted cells.

It was previously unclear how EGF co-stimulation could increase FGF-induced PC12 differentiation. Notably, depletion of FRS3 resulted in a much larger inhibition of neuronal differentiation after FGF and EGF costimulation as compared to the slight inhibition observed after stimulation with FGF alone. There are two likely consequences of FRS3 knockdown. First, loss of FRS3 
should disrupt FGF signal transduction. Second, since FRS3 can suppress EGF-induced activity of ERKs, depletion of FRS3 may enhance this activity, leading to amplification of the FRS2-ERKs negative feedback loop and a stronger inhibitory effect of EGF on the FGF signal pathway. Whether other mechanisms underlie the inhibition of differentiation in FRS3-depleted PC12 cells after EGF/FGF co-stimulation remains unknown. Further experiments, such as comparing the effects of FRS2 and FRS3 knockdown separately and in combination, should be performed to clarify the exact role of FRS3 in EGF/ FGF co-stimulation. Our observation suggests that FRS3 plays an important and complex role in cell signaling, especially under physiological conditions in which the cell encounters multiple stimuli.

FRS2 is not simply a link to the MAPK cascade but is a pivotal signal organizer. PY of FRS2 leads to Grb2mediated complex formation with Gab1, resulting in the recruitment and activation of PI3-kinase [2, 7]. VEGF treatment leads to the recruitment of Nck, p21-activated kinase, Crk, Grb2, and protein kinase C to FRS2 [10]. FRS2 serves as an alternative link of FGFRs to PKC activation in the central nervous system [39]. Most importantly, the embryos of FGFR1 $(\Delta)$ FRS/ $\Delta$ FRS mice, in which the FRS2-binding site on FGFR1 is deleted, die during late embryogenesis and exhibit defects in neural tube closure. Meanwhile, the mutant receptor can elicit FGFR1 functions during gastrulation and somitogenesis, as well as normal MAPK responses to FGF [40]. Recent reports described several atypical mechanisms underlying FRS2-mediated signal transduction. FGF-induced phosphorylation of FRS2 causes departure of Cks1 from FRS2, leading to the ubiquitination and degradation of p27(Kip1), which results in the activation of the cyclin E/A-Cdk complex [41]. Similarly, GTPase Rnd1 is released from the phosphorylated FRS2 after FGF treatment and suppresses RhoA activity [42]. The role of PY of FRS2 in these processes has not yet been reported. So far, there are only limited reports concerning EGF/ FGF double stimulation, as FGF and EGF share several common downstream pathways. This study, in which we report attenuation of FGF-induced PY of FRS2 by EGF co-stimulation and a possible mechanism underlying this phenomenon, sheds new light on FRS2-mediated regulation of the FGF pathway under physiological conditions (Supplementary information, Figure S4).

\section{Materials and Methods}

\section{Plasmids and antibodies}

Constructs encoding GST fusion proteins of FRS2 deletion mutants were generated by subcloning the fragments in frame into the pGEX5X-3 vector (Amersham Pharmacia Biotech). Site muta- genesis of FRS2 was performed using QuickChange site-directed mutagenesis kits (Stratagene), according to the manufacturer's instructions. FPA, FPB, and FRS2-3KL eukaryotic expressing plasmids were generated by inserting the appropriate fragment into the pRK5-RS vector. Rabbit polyclonal antibodies against FRS2 were generated against the GST fusion proteins of full-length FRS2 or its C-terminal. Anti-ERK1/2 and anti-Grb2 antibodies were purchased from Santa Cruz. Anti-HA monoclonal antibody, antiShp2 antibody, and anti-phospho-tyrosine antibody (4G10) were acquired from Upstate Biotech. Anti-phospho-MAPK, anti-pTP, anti-Akt, and anti-phospho-Akt antibodies were purchased from Cell Signaling Technology. Secondary antibodies were obtained from Bio-Rad.

\section{Cell lysis, immunoprecipitation, association with fusion} proteins, and immunoblotting

Prior to lysis, cells grown to $80 \%-90 \%$ confluency were treated with inhibitors and agonists as indicated. Next, they were washed once with phosphate-buffered saline and then placed on ice in lysis buffer containing $50 \mathrm{mM}$ HEPES (pH 7.5), $150 \mathrm{mM} \mathrm{NaCl}$, $1 \%$ Triton $\mathrm{X}-100,1 \mathrm{mM}$ EDTA, $10 \%$ glycerol, $10 \mathrm{mM}$ sodium pyrophosphate, $2 \mathrm{mM}$ sodium orthovanadate, $10 \mathrm{mM}$ sodium fluoride, $1 \mathrm{mM}$ phenylmethylsulfonyl fluoride, and $10 \mu \mathrm{g} / \mathrm{ml}$ aprotinin. The lysates were precleared by centrifugation at $13000 \mathrm{rpm}$ for $10 \mathrm{~min}$ at $4{ }^{\circ} \mathrm{C}$. The supernatants were subsequently subjected to immunoprecipitation using the indicated antibodies and $20 \mu \mathrm{l}$ of Protein A-Sepharose for $4 \mathrm{~h}$ at $4{ }^{\circ} \mathrm{C}$. Alternatively, the lysates were subjected to in vitro associations with either $3 \mu \mathrm{g}$ of GST-FRS2 fusion protein or GST alone prebound to $10 \mu \mathrm{l}$ of gluthathioneagarose beads. The precipitates were washed thrice with $1 \mathrm{ml}$ of HNTG buffer, suspended in SDS sample buffer, boiled for $10 \mathrm{~min}$, and subjected to gel electrophoresis on a $10 \%$ gel. Following SDSPAGE, the proteins were transferred to a nitrocellulose membrane and subsequently immunoblotted.

\section{Generation of recombinant retrovirus and cell infection}

BOSC23 package cells were transfected with pMSCV plasmids. At $48 \mathrm{~h}$ after transfection, the media containing virus particles were collected and filtered through a $0.45-\mu \mathrm{m}$ syringe filter. The retroviruses were stored at $-20{ }^{\circ} \mathrm{C}$ before infection. At $12 \mathrm{~h}$ before infection, $5 \times 10^{4}$ cells were plated on a well of six-well dishes. For infection, the cells were cultured in a mixture of $1 \mathrm{ml}$ retrovirus, $4 \mu \mathrm{g} / \mathrm{ml}$ polybrene, and $1 \mathrm{ml}$ complete DMEM medium at $37{ }^{\circ} \mathrm{C}$ for $12 \mathrm{~h}$, and the infection efficiency was observed by detecting green fluorescence.

\section{PC12 cell differentiation assays}

PC12 cells were infected with empty retrovirus or retroviruses carrying the FRS2-WT or FRS2-3KL $24 \mathrm{~h}$ before induction. In some experiments, cells were transfected with siRNAs using Lipofectamine2000 $48 \mathrm{~h}$ before induction. The cells were then grown in DMEM medium supplemented with $2 \%$ horse serum plus $1 \%$ FCS and then FGF, EGF, or FGF plus EGF was added. Neurite outgrowth was scored and quantified 48-72 h later under the microscope. Cells exhibiting a dendrite length twice that of the cell body were considered differentiated.

\section{Sequences for FRS3 siRNAs and RT-PCR primers}

siRNAs for FRS3 as well as the negative control were designed 
and manufactured from Shanghai GenePharma Co., Ltd. The sequences were as follows: FRS3Ri313: 5'-GCA GUG UAA CAG CAU CAA UTT-3'; FRS3Ri1188: 5'-CAA GGG UCU UCA ACU UUG ATT-3'; FRS3Ri1366: 5'-CGU GAU UGA CCU CAA GAA ATT-3'; NC: 5'-UUC UCC GAA CGU GUC ACG UTT-3'. The sequences for RT-PCR were as follows: forward primer of FRS3: 5'-GCA CCT GCA CCA GCA GG-3'; reverse primer of FRS3: 5'CAC TGA CAT TCT CAT AGG TG-3'; forward primer of GAPDH: 5'-TGT TGC CAT CAA TGA CCC CTT-3'; reverse primer of GAPDH: 5'-CTC CAC GAC GTA CTC AGC G-3'.

\section{Acknowledgments}

This research was supported by grants from National Natural Science Foundation of China (No. 30730055 and 30623002), National Basic Research Program of China (No. 2007CB914504), the National High Technology and Development Program of China (No. 2006AA021308), the Program of Shanghai Subject Chief Scientist (No. 08XD14051), and the Chinese Academy of Sciences (No. KSCX2-YW-R-108 and KSCX1-YW-R-67).

\section{References}

1 Kouhara H, Hadari YR, Spivak-Kroizman T, et al. A lipid-anchored Grb2-binding protein that links FGF-receptor activation to the Ras/MAPK signaling pathway. Cell 1997; 89:693702.

2 Hadari YR, Gotoh N, Kouhara H, et al. Critical role for the docking-protein FRS2 alpha in FGF receptor-mediated signal transduction pathways. Proc Natl Acad Sci USA 2001; 98:8578-8583.

3 Hadari YR, Kouhara H, Lax I, et al. Binding of Shp2 tyrosine phosphatase to FRS2 is essential for fibroblast growth factor-induced PC12 cell differentiation. Mol Cell Biol 1998; 18:3966-3973.

4 Ong SH, Guy GR, Hadari YR, et al. FRS2 proteins recruit intracellular signaling pathways by binding to diverse targets on fibroblast growth factor and nerve growth factor receptors. Mol Cell Biol 2000; 20:979-989.

5 Wang JK, Xu H, Li HC, et al. Broadly expressed SNT-like proteins link FGF receptor stimulation to activators of Ras. Oncogene 1996; 13:721-729.

$6 \mathrm{Xu} \mathrm{H}$, Lee KW and Goldfarb M. Novel recognition motif on fibroblast growth factor receptor mediates direct association and activation of SNT adapter proteins. J Biol Chem 1998; 273:17987-17990.

7 Ong SH, Hadari YR, Gotoh N, et al. Stimulation of phosphatidylinositol 3-kinase by fibroblast growth factor receptors is mediated by coordinated recruitment of multiple docking proteins. Proc Natl Acad Sci USA 2001; 98:6074-6079.

8 Melillo RM, Santoro M, Ong SH, et al. Docking protein FRS2 links the protein tyrosine kinase RET and its oncogenic forms with the mitogen-activated protein kinase signaling cascade. Mol Cell Biol 2001; 21:4177-4187.

9 Yan KS, Kuti M, Yan S, et al. FRS2 PTB domain conformation regulates interactions with divergent neurotrophic receptors. J Biol Chem 2002; 277:17088-17094.

10 Stoletov KV, Ratcliffe KE, Terman BI. Fibroblast growth factor receptor substrate 2 participates in vascular endothelial growth factor-induced signaling. FASEB J 2002; 16:12831285.

11 Dhalluin C, Yan KS, Plotnikova O, et al. Structural basis of SNT PTB domain interactions with distinct neurotrophic receptors. Mol Cell 2000; 6:921-929.

12 Wong A, Lamothe B, Lee A, et al. FRS2 alpha attenuates FGF receptor signaling by Grb2-mediated recruitment of the ubiquitin ligase Cbl. Proc Natl Acad Sci USA 2002; 99:66846689.

13 Guy G R, Yusoff P, Bangarusamy D, et al. Dockers at the crossroads. Cell Signal 2002; 14:11-20.

14 Lax I, Wong A, Lamothe B, et al. The docking protein FRS2alpha controls a MAP kinase-mediated negative feedback mechanism for signaling by FGF receptors. Mol Cell 2002; 10:709-719.

$15 \mathrm{Wu}$ Y, Chen Z, Ullrich A. EGFR and FGFR signaling through FRS2 is subject to negative feedback control by ERK1/2. Biol Chem 2003; 384:1215-1226.

16 Gutkind JS. Regulation of mitogen-activated protein kinase signaling networks by G protein-coupled receptors. Sci STKE 2000; 2000:RE1.

17 Chang L, Karin M. Mammalian MAP kinase signalling cascades. Nature 2001; 410:37-40.

18 Chang F, Steelman LS, Lee JT, et al. Signal transduction mediated by the Ras/Raf/MEK/ERK pathway from cytokine receptors to transcription factors: potential targeting for therapeutic intervention. Leukemia 2003; 17:1263-1293.

19 Hogan BL. Morphogenesis. Cell 1999; 96:225-233.

20 Pawson T, Saxton TM. Signaling networks--do all roads lead to the same genes? Cell 1999; 97:675-678.

21 Schlessinger J. Cell signaling by receptor tyrosine kinases. Cell 2000; 103:211-225.

22 Vaudry D, Stork PJ, Lazarovici P, et al. Signaling pathways for PC12 cell differentiation: making the right connections. Science 2002; 296:1648-1649.

23 Seger R, Krebs EG. The MAPK signaling cascade. FASEB J 1995; 9:726-735.

24 Gonzalez FA, Raden DL, Davis RJ. Identification of substrate recognition determinants for human ERK1 and ERK2 protein kinases. J Biol Chem 1991; 266:22159-22163.

25 Jacobs D, Glossip D, Xing H, et al. Multiple docking sites on substrate proteins form a modular system that mediates recognition by ERK MAP kinase. Genes Dev 1999; 13:163-175.

26 Tanoue T, Adachi M, Moriguchi T, et al. A conserved docking motif in MAP kinases common to substrates, activators and regulators. Nat Cell Biol 2000; 2:110-116.

27 Fantz DA, Jacobs D, Glossip D, et al. Docking sites on substrate proteins direct extracellular signal-regulated kinase to phosphorylate specific residues. J Biol Chem 2001; 276:27256-27265.

28 Rabin SJ, Cleghon V, Kaplan DR. SNT, a differentiationspecific target of neurotrophic factor-induced tyrosine kinase activity in neurons and PC12 cells. Mol Cell Biol 1993; 13:2203-2213.

29 Huang L, Gotoh N, Zhang S, et al. SNT-2 interacts with ERK2 and negatively regulates ERK2 signaling in response to EGF stimulation. Biochem Biophys Res Commun 2004; 324:1011-1017.

30 Huang L, Watanabe M, Chikamori M, et al. Unique role of 
SNT-2/FRS2beta/FRS3 docking/adaptor protein for negative regulation in EGF receptor tyrosine kinase signaling pathways. Oncogene 2006; 25:6457-6466.

31 Tanoue T, Maeda R, Adachi M, et al. Identification of a docking groove on ERK and p38 MAP kinases that regulates the specificity of docking interactions. EMBO J 2001; 20:466479.

32 Zhou T, Sun L, Humphreys J, et al. Docking interactions induce exposure of activation loop in the MAP kinase ERK2. Structure 2006; 14:1011-1019.

33 Hotamisligil GS, Peraldi P, Budavari A, et al. IRS-1-mediated inhibition of insulin receptor tyrosine kinase activity in TNFalpha- and obesity-induced insulin resistance. Science 1996; 271:665-668.

34 Ricort JM, Tanti JF, Van Obberghen E, et al. Different effects of insulin and platelet-derived growth factor on phosphatidylinositol 3-kinase at the subcellular level in 3T3-L1 adipocytes. A possible explanation for their specific effects on glucose transport. Eur J Biochem 1996; 239:17-22.

35 Fujishiro M, Gotoh Y, Katagiri H, et al. Three mitogenactivated protein kinases inhibit insulin signaling by different mechanisms in 3T3-L1 adipocytes. Mol Endocrinol 2003; 17:487-497.

36 Kao S, Jaiswal RK, Kolch W, et al. Identification of the mechanisms regulating the differential activation of the mapk cascade by epidermal growth factor and nerve growth factor in PC12 cells. J Biol Chem 2001; 276:18169-18177.

37 Bar-Sagi D, Feramisco JR. Microinjection of the ras oncogene protein into PC12 cells induces morphological differentiation. Cell 1985; 42:841-848.

38 Noda M, Ko M, Ogura A, et al. Sarcoma viruses carrying ras oncogenes induce differentiation-associated properties in a neuronal cell line. Nature 1985; 318:73-75.

39 Reuss B, von Bohlen und Halbach O. Fibroblast growth factors and their receptors in the central nervous system. Cell Tissue Res 2003; 313:139-157.

40 Hoch RV, Soriano P. Context-specific requirements for Fgfr1 signaling through Frs2 and Frs3 during mouse development. Development 2006; 133:663-673.

41 Zhang Y, Lin Y, Bowles C, et al. Direct cell cycle regulation by the fibroblast growth factor receptor (FGFR) kinase through phosphorylation-dependent release of $\mathrm{Cks} 1$ from FGFR substrate 2. J Biol Chem 2004; 279:55348-55354.

42 Harada A, Katoh H, Negishi M. Direct interaction of Rnd1 with FRS2 beta regulates Rnd1-induced down-regulation of RhoA activity and is involved in fibroblast growth factorinduced neurite outgrowth in PC12 cells. J Biol Chem 2005; 280:18418-18424.

(Supplementary information is linked to the online version of the paper on the Cell Research website.) 